\title{
Optimising the design of a limited-stop bus service for a branching network
}

\section{Hu Zhang MSC}

PhD Candidate, College of Transportation, Jilin University, Changchun, China

Shuzhi Zhao PhD

Professor, College of Transportation, Jilin University, Changchun, China

\author{
Huasheng Liu PhD \\ Lecturer, College of Transportation, Jilin University, Changchun, China \\ (corresponding author: liuhuasheng521@163.com) \\ Shidong Liang MSC \\ PhD Candidate, College of Transportation, Jilin University, Changchun, China
}

This paper presents an optimisation model for designing limited-stop lines for a branching bus network composed of several feeder lines and a trunk line. On the basis of the different demand patterns in the feeder lines and the trunk line, passenger assignment in the bus route is considered. The optimisation model is used to calculate the total cost of a transit system in which limited-stop services are implemented on bus routes. The total cost includes the cost of passenger waiting time and in-vehicle time, as well as the operator cost for each bus route. With better service planning of limited-stop lines, based on the demand behaviour in high-demand corridors, the total cost can be reduced (compared with the normal service costs of stopping all buses at all stops). A genetic algorithm is adopted to ascertain the optimal model. This algorithm can determine continuous and discrete optimisation variables: the optimal bus frequencies and the best pattern of limited-stop use for each bus route. Finally, a numerical example is optimised. The results show that a limited-stop service for branching corridors can reduce the total cost of the transit system.

\section{Notation}

C total cost of a branching corridor

$C^{l_{k}} \quad$ total cost in the feeder line $l_{k}$

$F \quad$ limited operable fleet size for the branching corridor

$f_{\mathrm{A}}^{L_{k}} \quad$ frequency of fleet $\mathrm{A}$ in the trunk line $L_{k}$

$n_{l_{k}}$

$R_{i}$

$\mathrm{TD}_{\mathrm{A}}^{l_{k}}$

$\mathrm{TD}_{\mathrm{B}}^{l_{k}}$

$\mathrm{TI}_{\mathrm{A}}^{l_{k}}$

$\mathrm{TI}_{\mathrm{B}}^{l_{k}}$

$\mathrm{TR}^{l_{k}}$

$\mathrm{TW}^{l_{k}}$

$V_{\mathrm{A}, i}^{l_{k}+}$

$V_{\mathrm{A}, i}^{l_{k}-}$

$V_{\mathrm{AB}, i}^{l_{k}+}$

$V_{\mathrm{AB}, i}^{l_{k}-}$

frequencies of fleet $A$

frequencies of fleet $B$

number of stations in the feeder line $l_{k}$

bus running time between stations $i$ and $i+1$

average dwelling time of fleet $\mathrm{A}$ at station $i$

average dwelling time of fleet $\mathrm{B}$ at station $i$

in-vehicle time of passengers riding fleet $A$

in-vehicle time of passengers riding fleet $\mathrm{B}$

running time of buses

total waiting time in the feeder line $l_{k}$

boarding of passengers that can only ride fleet $A$

buses at station $i$

alighting rate of passengers that can only ride fleet $\mathrm{A}$ buses at station $i$

boarding rate of passengers that have the opportunity to select fleet A or fleet B buses

alighting rate of passengers that have the opportunity to select fleet A or fleet B buses

demand from station $i$ in the feeder line $l_{k}$ to $j$ in the entire bus route

type of station $i$, which equals 1 if the station is serviced by fleet $\mathrm{B}$ and 0 otherwise

value of in-vehicle time

value of running time

value of waiting time

\section{Introduction}

In public transit systems with high demand levels due to the fluctuating demand patterns and an unbalanced distribution of passengers along the corridor, the use of a normal all-stop service is not adequate and will not guarantee efficient operation. However, by adjusting the frequency of services and by establishing limited stops, these limited-stop services appear to be an efficient alternative operational strategy which could improve the service level of a transit system. By skipping a subset of stops, the limited-stop services can reduce passenger travel time and shorten the running time of buses. This will enable the transit operator to utilise fewer vehicles while still meeting the same demand along the corridor. Given the advantages of limited-stop services in a bus route, similar services should be extended to the design of the transit network, in order to improve the service level of multiple bus lines.

Designing limited-stop services as an optimisation strategy for a bus line or corridor has been studied by a number of authors in an attempt to select the appropriate limited stops and determine the bus frequencies. Afanasiev and Liberman (1982) stated that bus services consist of three types of services: normal, limited stop and express. The authors of this study also addressed the design of skipped-stop lines. The beginning and the end of a limited-stop line are determined based on a model designed to fulfil passenger demand but with frequency constraint. The experience in the city of Chimkent shows that passenger travel time and gasoline consumption both decrease considerably when using a limited-stop service, compared with the equivalent all-stop services. Silverman (1998) analysed the 
characteristics of limited-stop services and recognised that such services can improve bus speed and reliability. Passenger response to this limited-stop service strategy is also described in the Silverman (1998) study. Tétreault and Ahmed (2010) proposed a model to determine the skipped stops and estimated the running times for a limited-stop line in parallel with a normal line. The objective of this study was to maximise the running time savings of buses and to reduce the waiting time of passengers that arrive at stops. The numerical results show that implementing limited-stop lines can reduce the running time. Leiva et al. (2010) addressed the design of limited-stop services with capacity constraints and determined the optimum frequencies of different types of buses. The methodology of Leiva et al. (2010) minimises the sum of wait time, travel time, transfer time and operator costs. Given the effect of vehicle capacity constraints and transfers, the proposed method optimises the frequencies, type of vehicles and serving stations. Cortés et al. (2011) proposed integrating short-turning and deadheading services. Short-turning and deadheading services are designed for a specific, single line (in a two-line system), with an all-stop service implemented on the other line. The model determines the frequencies and serving zones of two bus fleets, thus providing a short-turn strategy. Sun et al. (2013) analysed the travel time model under mixed traffic conditions and proposed an optimisation model for designing skip-stop services that can minimise the total travel time for both car travellers and bus travellers.

With regard to the bus frequency design aspect of a network, Sun and Mark (2010) analysed the effects of different scheduling alternatives on a branching transit route. The passenger travelling time in the trunk and feeder line is examined under a variety of schedule alternatives that could be implemented. These schedule alternatives are related to the assignment of bus to trunk-feeder lines and bus holding strategies. The objective is to estimate the performance of a branching transit route with different scheduling alternatives. Yu et al. (2010) proposed a bi-level model to determine the frequency of an urban public transportation system using a genetic algorithm with fleet size constraint. Passenger assignment in the bus routes was considered. Additionally, the total travel time, integrating waiting time and in-vehicle time of passengers, was minimised. In a real transit network, optimisation can improve the overall service level. Sivakumaran et al. (2012) analysed the schedule coordination properties that can save costs in a trunk-feeder transit system. The result is that the total costs of both the user and the operator can be reduced by coordinating the schedules of trunkfeeder vehicles. Dell'Olio et al. (2012) optimised the frequency and bus size in a transit network. This study proposed a bi-level optimisation model with capacity constraints, to assign passengers to different bus routes. Then, the model is able to minimise the total cost of the user and the operator by determining the optimised frequency and bus sizes. Along these lines, an optimisation model with constraints on bus capacity (in terms of bus size and setting frequencies on each route in the system) has been proposed in accordance with the premises detailed below.

In this paper, to implement a limited-stop service on the network, this paper proposes an optimisation method for the design of limited-stop services for a branching corridor. A branching corridor denotes a simple feeder-trunk network. Assume the limited-stop service is implemented on each bus route, and the total cost of the transit system in the feeder lines and trunk line is calculated. By minimising the total cost, the optimal operational scheme in the feeder line and trunk line (and the optimal frequency) can be found. The total cost is calculated in terms of waiting time, in-vehicle travel time and operator cost (Baaj and Mahmassani, 1995; Ceder and Wilson, 1986; LeBlanc (1988); Mauttone and Urquhart, 2009).

\section{Optimisation modelling}

In this paper, the idealised branching corridor is a tree-shaped network in one direction, as shown in Figure 1. The network consists of a trunk line and several feeder lines. Each feeder line (where only one bus route is provided) connects to the trunk line. The bus route provides service from uptown to downtown (central business district (CBD)), first along the feeder line and then the trunk line. In this approach, suppose now that the trunk line is disconnected from the junction notes between the feeder lines and trunk line, denoted as $L_{1}, L_{2}, \ldots$, and the feeder lines are denoted as $l_{1}, l_{2}, \ldots$. This paper further considers that the single bus route is operated in the feeder line, while buses of different routes flow into the trunk line. Then, limited-stop schemes of the single bus route are determined in both the feeder line and the trunk line, respectively.

\subsection{Cost of feeder-line service}

In a branching corridor, a limited-stop service for a bus line is provided by buses of fleet $\mathrm{A}$ and fleet $\mathrm{B}$. The fleet $\mathrm{A}$ buses serve every station along the bus route, while the fleet B buses serve specifically designated stations where the passengers can

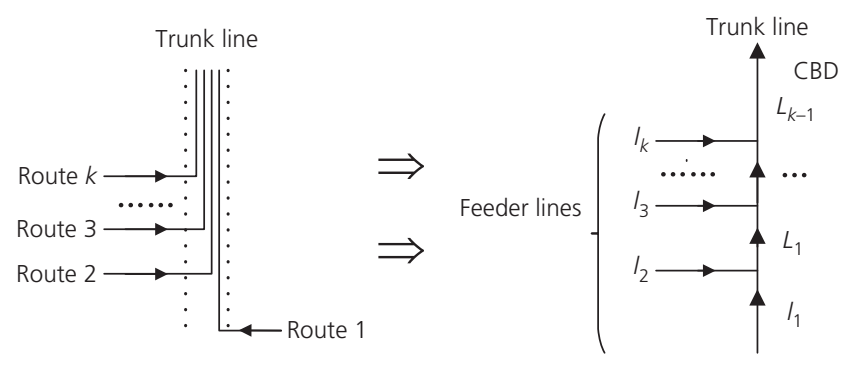

Figure 1. Sketch of branching corridors 
either board or get off the buses. Therefore, the passengers will board the first arriving vehicle which can serve their specific origin-destination trips and which can complete that trip without the need for transfers. This process closely reflects the travel habits of the passengers. The limited-stop scheme of a feeder line is shown in Figure 2.

In a feeder line, passengers can only board stations without the services of fleet B buses. Additionally, passengers have a choice of bus fleets at stations served by both A and B buses. Thus, given an origin-destination trip matrix and the types of stations on one bus route that operates along both the feeder line and trunk line, the passenger boarding and alighting rate at station $i$ in feeder line $l_{k}$ can be shown as follows

1.

$$
\begin{gathered}
V_{\mathrm{A}, i}^{l_{k}+}=\sum_{j} v_{(i, j)}^{l_{k}} \max \left[\left(1-x_{i}^{l_{k}}\right),\left(1-x_{j}^{l_{k}}\right)\right] \quad \forall j ; \\
\forall i=1,2, \ldots, n_{l_{k}}
\end{gathered}
$$

2.

$$
\begin{gathered}
V_{\mathrm{A}, i}^{l_{k}-}=\sum_{i} v_{(i, j)}^{l_{k}} \max \left[\left(1-x_{i}^{l_{k}}\right),\left(1-x_{j}^{l_{k}}\right)\right] \\
\forall i=1,2, \ldots, n_{l_{k}} ; j \leq i
\end{gathered}
$$

3. $\quad V_{\mathrm{AB}, i}^{l_{k}+}=\sum_{j} v_{(i, j)}^{l_{k}} x_{i}^{l_{k}} x_{j}^{l_{k}} \quad \forall j ; \quad \forall i=1,2, \ldots, n_{l_{k}}$
4. $\quad V_{\mathrm{AB}, i}^{l_{k}-}=\sum_{i} v_{(i, j)}^{l_{k}} x_{i}^{l_{k}} x_{j}^{l_{k}} \quad \forall i=1,2, \ldots, n_{l_{k}} ; j \leq i$

where $V_{\mathrm{A}, i}^{l_{k}+}$ and $V_{\mathrm{A}, i}^{l_{k}-}$ are the boarding and alighting rate of passengers that can only ride the fleet $\mathrm{A}$ buses at station $i$, while $V_{\mathrm{AB}, i}^{l_{k}+}$ and $V_{\mathrm{AB}, i}^{l_{k}-}$ are the boarding and alighting rate of passengers that have a choice between fleet $\mathrm{A}$ and fleet $\mathrm{B}$. The probability that the line will be boarded is the ratio of its frequency divided by the combined frequency; $n_{l_{k}}$ is the number of stations in the feeder line $l_{k} ; f_{\mathrm{A}}^{l_{k}}$ and $f_{\mathrm{B}}^{l_{k}}$ are the frequencies of fleet $\mathrm{A}$ and fleet $\mathrm{B} ; x_{i}^{l_{k}}$ indicates the type of station $i$, which

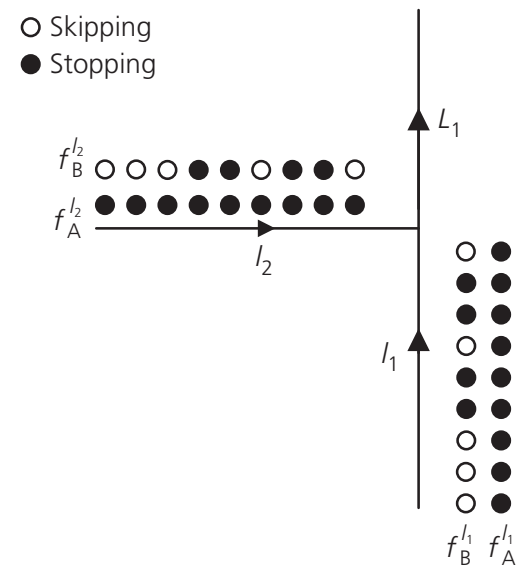

Figure 2. Scheme of limited-stop service in feeder line

equals 1 if the station is served by fleet B and 0 otherwise; $v_{(i, j)}^{l_{k}}$ is the demand from station $i$ in the feeder line $l_{k}$ to $j$ in the entire bus route.

The average waiting time at station $i$, the headway of buses, is the inverse of service frequency. For the station that is serviced by fleet $\mathrm{A}$, the average waiting time is $1 / f_{\mathrm{A}}^{l_{k}}$, while for the station that is serviced by both fleets $\mathrm{A}$ and $\mathrm{B}$, the average waiting time is $1 /\left(f_{\mathrm{A}}^{l_{k}}+f_{\mathrm{B}}^{l_{k}}\right)$. The total waiting time in feeder line $l_{k}$ is

5. $\quad \mathrm{TW}^{l_{k}}=\sum_{i}^{n_{l_{k}}}\left[\frac{1}{f_{\mathrm{A}}^{l_{k}}} V_{\mathrm{A}, i}^{l_{k}+}+\frac{1}{f_{\mathrm{A}}^{l_{k}}+f_{\mathrm{B}}^{l_{k}}} V_{\mathrm{AB}, i}^{l_{k}+}\right]$

As regards in-vehicle time, this paper considers two components (dwelling time and travel time). The average dwelling time of fleets $\mathrm{A}$ and $\mathrm{B}$ at station $i$, denoted as $\mathrm{TD}_{\mathrm{A}}^{l_{k}}$ and $\mathrm{TD}_{\mathrm{B}}^{l_{k}}$, is based on the number of boarding and alighting passengers. When buses dwell on the station $i$, the number of passengers in the vehicle of fleet $\mathrm{A}$ is equal to $\left(V_{\mathrm{A}, i-1}^{l_{k}+}+\right.$ $\left.\left(f_{\mathrm{A}}^{l_{k}} /\left(f_{\mathrm{A}}^{l_{k}}+f_{\mathrm{B}}^{l_{k}}\right)\right) V_{\mathrm{AB}, i-1}^{l_{k}+}-V_{\mathrm{A}, i}^{l_{k}-}-\left(f_{\mathrm{A}}^{l_{k}} /\left(f_{\mathrm{A}}^{l_{k}}+f_{\mathrm{B}}^{l_{k}}\right)\right) V_{\mathrm{B}, i}^{l_{k}-}\right)$. The travel time between stations, denoted as $R_{i}$, is the running time of buses from station $i$ to $i+1$. Thus, the in-vehicle time of passengers riding fleet $\mathrm{A}$ can be expressed by
6.

$$
\begin{aligned}
\mathrm{TI}_{\mathrm{A}}^{l_{k}}= & \sum_{i=2}^{n_{l_{k}}}\left\{\left(V_{\mathrm{A}, i-1}^{l_{k}+}+\frac{f_{\mathrm{A}}^{l_{k}}}{f_{\mathrm{A}}^{l_{k}}+f_{\mathrm{B}}^{l_{k}}} V_{\mathrm{AB}, i-1}^{l_{k}+}-V_{\mathrm{A}, i}^{l_{k}-}-\frac{f_{\mathrm{A}}^{l_{k}}}{f_{\mathrm{A}}^{l_{k}}+f_{\mathrm{B}}^{l_{k}}} V_{\mathrm{AB}, i}^{l_{k}-}\right) \cdot \mathrm{TD}_{\mathrm{A}, i}^{l_{k}}\right\} \\
& +\sum_{i=1}^{n_{l_{k}}}\left\{\left(V_{\mathrm{A}, i}^{l_{k}+}+\frac{f_{\mathrm{A}}^{l_{k}}}{f_{\mathrm{A}}^{l_{k}}+f_{\mathrm{B}}^{l_{k}}} V_{\mathrm{AB}, i}^{l_{k}+}-V_{\mathrm{A}, i}^{l_{k}-}-\frac{f_{\mathrm{A}}^{l_{k}}}{f_{\mathrm{A}}^{l_{k}}+f_{\mathrm{B}}^{l_{k}}} V_{\mathrm{AB}, i}^{l_{k}-}\right) \cdot R_{i}\right\}
\end{aligned}
$$


7.

$$
\begin{aligned}
& \operatorname{TD}_{\mathrm{A}, i}^{l_{k}}=\max {\left[a \cdot\left(\frac{1}{f_{\mathrm{A}}^{l_{k}}} V_{\mathrm{A}, i}^{l_{k}+}+\frac{1}{f_{\mathrm{A}}^{l_{k}}+f_{\mathrm{B}}^{l_{k}}} V_{\mathrm{AB}, i}^{l_{k}+}\right),\right.} \\
&\left.b \cdot\left(\frac{1}{f_{\mathrm{A}}^{l_{k}}} V_{\mathrm{A}, i}^{l_{k}-}+\frac{1}{f_{\mathrm{A}}^{l_{k}}+f_{\mathrm{B}}^{l_{k}}} V_{\mathrm{AB}, i}^{l_{k}-}\right)\right]
\end{aligned}
$$

Here, $a$ and $b$ are the average passenger boarding and alighting times; $R_{i}$ is the bus running time between stations $i$ and $i+1$. Similarly, the in-vehicle time of passengers riding fleet $B$ can be expressed by

8.

$$
\begin{aligned}
\mathrm{TI}_{\mathrm{B}}^{l_{k}}= & \sum_{i=2}^{n_{l_{k}}}\left\{\left(\frac{f_{\mathrm{B}}^{l_{k}}}{f_{\mathrm{A}}^{l_{k}}+f_{\mathrm{B}}^{l_{k}}} V_{\mathrm{AB}, i-1}^{l_{k}+}-\frac{f_{\mathrm{B}}^{l_{k}}}{f_{\mathrm{A}}^{l_{k}}+f_{\mathrm{B}}^{l_{k}}} V_{\mathrm{AB}, i}^{l_{k}-}\right) \cdot \mathrm{TD}_{\mathrm{B}, i}^{l_{k}}\right\} \\
& +\sum_{i=1}^{n_{l_{k}}}\left\{\left(\frac{f_{\mathrm{B}}^{l_{k}}}{f_{\mathrm{A}}^{l_{k}}+f_{\mathrm{B}}^{l_{k}}} V_{\mathrm{AB}, i}^{l_{k}+}-\frac{f_{\mathrm{B}}^{l_{k}}}{f_{\mathrm{A}}^{l_{k}}+f_{\mathrm{B}}^{l_{k}}} V_{\mathrm{AB}, i}^{l_{k}-}\right) \cdot R_{i}\right\}
\end{aligned}
$$

Note that since the dwelling time at a skipped station is equal to 0 , the average dwelling time of fleet $\mathrm{B}$ can be expressed by

9. $\quad \mathrm{TD}_{\mathrm{B}, i}^{l_{k}}=x_{i} \cdot \max \left[a \cdot \frac{1}{f_{\mathrm{A}}^{l_{k}}+f_{\mathrm{B}}^{l_{k}}} V_{\mathrm{B}, i}^{l_{k}+}, b \cdot \frac{1}{f_{\mathrm{A}}^{l_{k}}+f_{\mathrm{B}}^{l_{k}}} V_{\mathrm{B}, i}^{l_{k}-}\right]$

The above formulae calculate the time that passengers spend on travel. For the operator, this paper considers the running time of buses, which is similar to the in-vehicle time of passengers. Operator running time can be calculated as follows

10. $\mathrm{TR}^{l_{k}}=f_{\mathrm{A}}^{l_{k}} \sum_{i=1}^{n_{l_{k}}}\left(R_{i}+\mathrm{TD}_{\mathrm{A}, i}^{l_{k}}\right)+f_{\mathrm{B}}^{l_{k}} \sum_{i=1}^{n_{l_{k}}}\left(R_{i}+\mathrm{TD}_{\mathrm{B}, i}^{l_{k}}\right)$

Finally, the total cost of feeder line $l_{k}$ is the variable time items multiplied by the value of time, accordingly

11. $C^{l_{k}}=\phi_{\mathrm{w}} \cdot \mathrm{TW}^{l_{k}}+\phi_{\mathrm{i}} \cdot\left(\mathrm{TI}_{\mathrm{A}}^{l_{k}}+\mathrm{TI}_{\mathrm{B}}^{l_{k}}\right)+\phi_{\mathrm{R}} \cdot \mathrm{TR}^{l_{k}}$

Here, $\phi_{\mathrm{w}}, \phi_{\mathrm{i}}$ and $\phi_{\mathrm{R}}$ are the value of waiting time, in-vehicle time and running time, respectively.

\subsection{Cost of a trunk-line service}

In a trunk line, multiple routes normally operate at the same stations (i.e. there are $k$ bus routes which serve the trunk line $L_{k}$ ). Passengers have more chances to select alternative bus routes in the trunk line than they have in the feeder line. Figure 3 shows a simple trunk-feeder transit system.

In the trunk line $L_{k}$, passenger boarding and alighting rates at station $i$ can be shown as follows

12. $V_{\mathrm{A}, i}^{L_{k}+}=\sum_{j} v_{(i, j)}^{l_{k}} \cdot \prod_{m=1}^{k-1} \max \left[\left(1-x_{i}^{l_{m}}\right),\left(1-x_{j}^{l_{m}}\right)\right]$
for $i=1,2, \ldots, n_{l_{k}}$

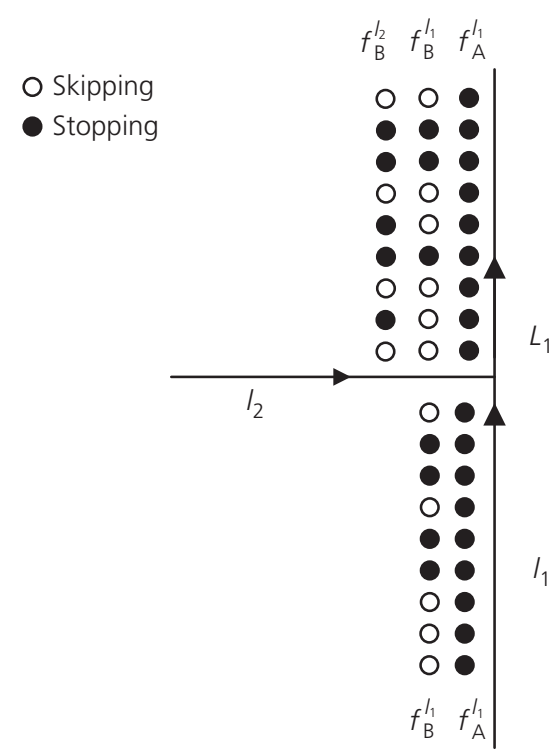

Figure 3. Scheme of limited-stop service in trunk line

13.

$$
\begin{gathered}
V_{\mathrm{A}, i}^{L_{k}-}=\sum_{i} v_{(j, i)}^{l_{k}} \cdot \prod_{m=1}^{k-1} \max \left[\left(1-x_{i}^{l_{m}}\right),\left(1-x_{j}^{l_{m}}\right)\right] \\
\quad \text { for } i=1,2, \ldots, n_{l_{k}}
\end{gathered}
$$

$$
V_{L_{k}, \overline{\mathrm{A}}, i}^{l_{r}+}=\sum_{j}\left[\frac{f_{\mathrm{A}}^{l_{k}}}{f_{\mathrm{A}}^{l_{k}}+\sum_{r=1}^{k-1} f_{\mathrm{B}}^{l_{r}} x_{i}^{l_{r}} x_{j}^{l_{r}}} v_{(i, j)}^{l_{k}} \cdot x_{i}^{l_{r}} x_{j}^{l_{r}}\right]
$$$$
\text { for } i=1,2, \ldots, n_{l_{k}}
$$

15.

$$
\begin{gathered}
V_{L_{k}, \overline{\mathrm{A}}, i}^{l_{r}-}=\sum_{i} \frac{f_{\mathrm{A}}^{l_{k}}}{f_{\mathrm{A}}^{l_{k}}+\sum_{r=1}^{k-1} f_{\mathrm{B}}^{l_{\mathrm{B}}} x_{i}^{l_{r}} x_{j}^{l_{r}}} v_{(j, i)}^{l_{k}} \cdot x_{i}^{l_{r}} x_{j}^{l_{r}} \\
\text { for } i=1,2, \ldots, n_{l_{k}}
\end{gathered}
$$

16.

$$
\begin{aligned}
V_{L_{k}, \mathrm{~A} \overline{\mathrm{B}}, i}^{l_{r}+}=\sum_{j}\left[\frac{f_{\mathrm{B}}^{l_{r}}}{f_{\mathrm{A}}^{l_{k}}+\sum_{r=1}^{k-1} f_{\mathrm{B}}^{l_{r}} x_{i}^{l_{r}} x_{j}^{l_{r}}} v_{(i, j)}^{l_{k}} \cdot x_{i}^{l_{r}} x_{j}^{l_{r}}\right] \\
\text { for } i=1,2, \ldots, n_{l_{k}}
\end{aligned}
$$

17.

$$
\begin{gathered}
V_{L_{k}, \mathrm{AB}, i}^{l_{r}-}=\sum_{i} \frac{f_{\mathrm{B}}^{l_{r}}}{f_{\mathrm{A}}^{l_{k}}+\sum_{r=1}^{k-1} f_{\mathrm{B}}^{l_{r}} x_{i}^{l_{r}} x_{j}^{l_{r}}} v_{(j, i)}^{l_{k}} \cdot x_{i}^{l_{r}} x_{j}^{l_{r}} \\
\text { for } i=1,2, \ldots, n_{l_{k}}
\end{gathered}
$$

Also

18. $f_{\mathrm{A}}^{l_{k}}=\sum_{m}^{k+1} f_{\mathrm{A}}^{l_{m}}$ 
Here, $f_{\mathrm{A}}^{l_{k}}, f_{\mathrm{A}}^{l_{r}}$ and $f_{\mathrm{B}}^{l_{r}}$ are the frequencies of fleet $\mathrm{A}$ in trunk line $L_{k}$, fleet $\mathrm{A}$ and fleet $\mathrm{B}$ on route $l_{m}$ starting in the feeder line $l_{r} ; V_{\mathrm{A}, i}^{L_{k}+}$ and $V_{\mathrm{A}, i}^{L_{k}-}$ are the boarding and alighting rates of passengers that can only ride the fleet $\mathrm{A}$ buses at station $i$, while $V_{L_{k}, \overline{\mathrm{A}} \mathrm{B}, i}^{l_{r}+}$ and $V_{L_{k}, \overline{\mathrm{A}} \mathrm{B}, i}^{l_{r}-}$ are the boarding and alighting rates of passengers that select fleet $\mathrm{A} ; V_{L_{k}, \mathrm{~A} \overline{\mathrm{B}}, i}^{l_{r}+}$ and $V_{L_{k}, \mathrm{~A} \overline{\mathrm{B}}, i}^{l_{r}-}$ are the boarding and alighting rates of passengers that select the fleet $\mathrm{B}$ buses of route $l_{r} ; n_{l_{k}}$ is the number of stations in trunk line $L_{k} ; v_{(i, j)}^{l_{k}}$ is the demand from station $i$ in trunk line $L_{k}$ to $j$ in the rest of the bus route along trunk line $L_{k}$.

With regard to waiting time, for the stations that are serviced by fleet $\mathrm{A}$, the average waiting time is $1 / f_{\mathrm{A}}^{l_{k}}$, while for the stations that are served by both fleets $\mathrm{A}$ and $\mathrm{B}$, the average waiting time is $1 /\left(f_{\mathrm{A}}^{l_{k}}+\sum_{r=1}^{k-1} f_{\mathrm{B}}^{l_{r}} x_{i}^{l_{r}} x_{j}^{l_{r}}\right)$ at station $i$. The total waiting time in the trunk line $L_{k}$ is
The in-vehicle time of passengers riding fleet $\mathrm{B}$ on route $l_{r}$ can be expressed by

23.

$$
\begin{aligned}
\mathrm{TI}_{\mathrm{B}}^{l_{r}}= & \sum_{i=2}^{n_{l_{k}}}\left[\left(V_{L_{k}, \mathrm{~A} \overline{\mathrm{B}}, i-1}^{l_{r}+}-V_{L_{k}, \mathrm{~A} \overline{\mathrm{B}}, i}^{l_{r}-}\right) \cdot \mathrm{TD}_{\mathrm{B}, i}^{l_{r}}\right] \\
& +\sum_{i=1}^{n_{l_{k}}}\left[\left(V_{L_{k}, \mathrm{~A} \overline{\mathrm{B}}, i}^{l_{r}-}-V_{L_{k}, \mathrm{~A}, i}^{l_{r}+}\right) \cdot R_{i}\right]
\end{aligned}
$$

The total in-vehicle time in trunk line $L_{k}$ can be as follows

24. $\quad \mathrm{TI}^{l_{k}}=\mathrm{TI}_{\mathrm{A}}^{l_{k}}+\sum_{r=1}^{k+1} \mathrm{TI}_{\mathrm{B}}^{l_{r}}$

19. $\quad \mathrm{TW}^{l_{k}}=\sum_{i}^{n_{l_{k}}}\left\{\frac{1}{f_{\mathrm{A}}^{l_{k}}} V_{\mathrm{A}, i}^{l_{k}+}+\sum_{r} \sum_{j}\left[\frac{1}{f_{\mathrm{A}}^{l_{k}}+\sum_{r=1}^{k-1} f_{\mathrm{B}}^{l_{r}} x_{i}^{l_{r}} x_{j}^{l_{r}}} v_{(j, i)}^{l_{k}} x_{i}^{l_{r}} x_{j}^{l_{r}}\right]\right\}$

With regard to the in-vehicle time of passengers, based on the number of boarding and alighting passengers, the average dwelling time of fleet $\mathrm{A}$, denoted as $\mathrm{TD}_{\mathrm{A}, i}^{l_{k}}$, and fleet $\mathrm{B}$ on route $l_{r}$, denoted as $\mathrm{TD}_{\mathrm{B}, i}^{l_{r}}$, can be expressed at station $i$ by
For the operator, the bus running time can be shown as follows

$$
\text { 25. } \quad \mathrm{TR}^{l_{k}}=f_{\mathrm{A}}^{l_{k}} \sum_{i=1}^{n_{l_{k}}}\left(R_{i}+\mathrm{TD}_{\mathrm{A}, i}^{l_{k}}\right)+\sum_{r}^{k+1} f_{\mathrm{B}}^{l_{r}} \sum_{i=1}^{n_{l_{k}}}\left(R_{i}+\mathrm{TD}_{\mathrm{B}, i}^{l_{r}}\right)
$$

$$
\begin{gathered}
\operatorname{TD}_{\mathrm{A}, i}^{l_{k}}=\max \left\{a \cdot\left(\frac{1}{f_{\mathrm{A}}^{l_{k}}} V_{\mathrm{A}, i}^{l_{k}+}+\sum_{r} \sum_{j}\left[\frac{1}{f_{\mathrm{A}}^{l_{k}}+\sum_{r=1}^{k-1} f_{\mathrm{B}}^{l_{r}} x_{i}^{l_{r}} x_{j}^{l_{r}}} v_{(j, i)}^{l_{k}} x_{i}^{l_{r}} x_{j}^{l_{r}}\right]\right),\right. \\
\left.b \cdot\left(\frac{1}{f_{\mathrm{A}}^{l_{k}}} V_{\mathrm{A}, i}^{l_{k}+}+\sum_{r} \sum_{j}\left[\frac{1}{f_{\mathrm{A}}^{l_{k}}+\sum_{r=1}^{k-1} f_{\mathrm{B}}^{l_{r}} x_{i}^{l_{r}} x_{j}^{l_{r}}} v_{(j, i)}^{l_{k}} x_{i}^{l_{r}} x_{j}^{l_{r}}\right]\right)\right\}
\end{gathered}
$$

21.

$$
\operatorname{TD}_{\mathrm{B}, i}^{l_{r}}=x_{i}^{l_{r}} \cdot \max \left\{a \cdot \sum_{r} \sum_{j}\left[\frac{1}{f_{\mathrm{A}}^{l_{k}}+\sum_{r=1}^{k-1} f_{\mathrm{B}}^{l_{r}} x_{i}^{l_{r}} x_{j}^{l_{r}}} v_{(j, i)}^{l_{k}} x_{i}^{l_{r}} x_{j}^{l_{r}}\right],\right.
$$

$$
\left.b \cdot \sum_{r} \sum_{j}\left[\frac{1}{f_{\mathrm{A}}^{l_{k}}+\sum_{r=1}^{k-1} f_{\mathrm{B}}^{l_{r}} x_{i}^{l_{r}} x_{j}^{l_{r}}} v_{(j, i)}^{l_{k}} x_{i}^{l_{r}} x_{j}^{l_{r}}\right]\right\}
$$

Then, considering the travel time between stations, the in-vehicle time of passengers riding fleet $A$ can be expressed by
Finally, the total cost of feeder line $L_{k}$ is the variable time items multiplied by value of time, accordingly

26. $C^{l_{k}}=\phi_{\mathrm{w}} \cdot \mathrm{TW}^{l_{k}}+\phi_{\mathrm{i}} \cdot \mathrm{TI}^{l_{k}}+\phi_{\mathrm{R}} \cdot \mathrm{TR}^{l_{k}}$ for $k \geq 2$

The objective function for the total cost of a branching corridor with $h$ bus routes can be expressed by

27. $\min C=\sum_{k=1}^{h} C^{l_{k}}+\sum_{k=1}^{h-1} C^{l_{k}}$

22.

$$
\begin{aligned}
\mathrm{TI}_{\mathrm{A}}^{l_{k}}= & \sum_{i=2}^{n_{l_{k}}}\left[\left(V_{\mathrm{A}, i-1}^{L_{k}+}+\sum_{r} V_{L_{k}, \overline{\mathrm{A}} \mathrm{B}, i-1}^{l_{r}+}-V_{\mathrm{A}, i}^{L_{k}-}-\sum_{r} V_{L_{k}, \overline{\mathrm{A}} \mathrm{B}, i}^{l_{r}-}\right) \cdot \mathrm{TD}_{\mathrm{A}, i}^{l_{k}}\right] \\
& +\sum_{i=1}^{n_{l_{k}}}\left[\left(V_{\mathrm{A}, i}^{L_{k}+}+\sum_{r} V_{L_{k}, \mathrm{~B}, i}^{l_{r}+}-V_{L_{k}, \overline{\mathrm{A}}, i}^{l_{r}+}-\sum_{r} V_{L_{k}, \overline{\mathrm{A}}, i}^{l_{r}-}\right) \cdot R_{i}\right]
\end{aligned}
$$


s.t.

28.

$$
\begin{array}{r}
F \geq \sum_{k=1}^{h}\left\{f_{\mathrm{A}}^{l_{k}}\left[\sum_{i=1}^{N^{l_{k}}}\left(R_{i}+\mathrm{TD}_{\mathrm{A}, i}^{l_{k}}\right)+\sum_{i=1}^{N^{l_{k}}}\left(R_{i}+\mathrm{TD}_{\mathrm{A}, i}^{l_{k}}\right)\right]\right. \\
\left.+f_{\mathrm{B}}^{l_{k}}\left[\sum_{i=1}^{N^{l_{k}}}\left(R_{i}+\mathrm{TD}_{\mathrm{B}, i}^{l_{k}}\right)+\sum_{i=1}^{N^{l_{k}}}\left(R_{i}+\mathrm{TD}_{\mathrm{B}, i}^{l_{r}}\right)\right]\right\}
\end{array}
$$

29. $f_{\mathrm{A}}^{l_{k}} \geq 0$

30. $f_{\mathrm{B}}^{l_{k}} \geq 0$

31. $x_{i}^{l_{k}} \in(0,1)$

Here, $F$ is the limited operable fleet size for the branching corridor. Equation 29 is a fleet size constraint which ensures that the optimal service frequency does not exceed the maximum service frequency.

\section{Solution methods}

This research now requires a solution algorithm for the objective function described in the previous sections. This solution algorithm will enable us to find the optimum limited stop scheme and the corresponding frequencies of buses.

Determining the optimal scheme of a limited-stop service where the type of station is a zero-one variable is a non-linear programming problem and is difficult to solve. Thus, the genetic algorithm that imitates the process of natural evolution is used to search the fittest chromosome, which is the same as the optimal scheme (see - e.g. Agrawal and Mathew, 2004; Fan and Machemehl, 2006). In this paper, a chromosome consists of the entire schemes of bus routes as

32.

$$
\begin{aligned}
& {\left[x_{1}^{l_{1}}, x_{2}^{l_{1}}, \ldots x_{N_{l_{1}}}^{l_{1}}, x_{1}^{l_{2}}, x_{2}^{l_{2}}, \ldots x_{N_{l_{2}}}^{l_{2}}, \ldots, x_{1}^{l_{k}},\right.} \\
& \left.x_{2}^{l_{k}}, \ldots, x_{i}^{l_{k}}, \ldots, x_{N_{l_{k}}}^{l_{k}}, \ldots, x_{1}^{l_{h}}, x_{2}^{l_{h}}, \ldots x_{N_{l_{h}}}^{l_{h}}\right]
\end{aligned}
$$

Here, $h$ is the number of bus routes in a branching corridor; $x_{i}^{l_{k}}$ is a zero-one variable that indicates a possible limited-stop type of station $i$ on route $l_{k} ; N_{l_{h}}$ is the number of stations on route $l_{h}$ that includes the stations in the feeder and trunk lines.

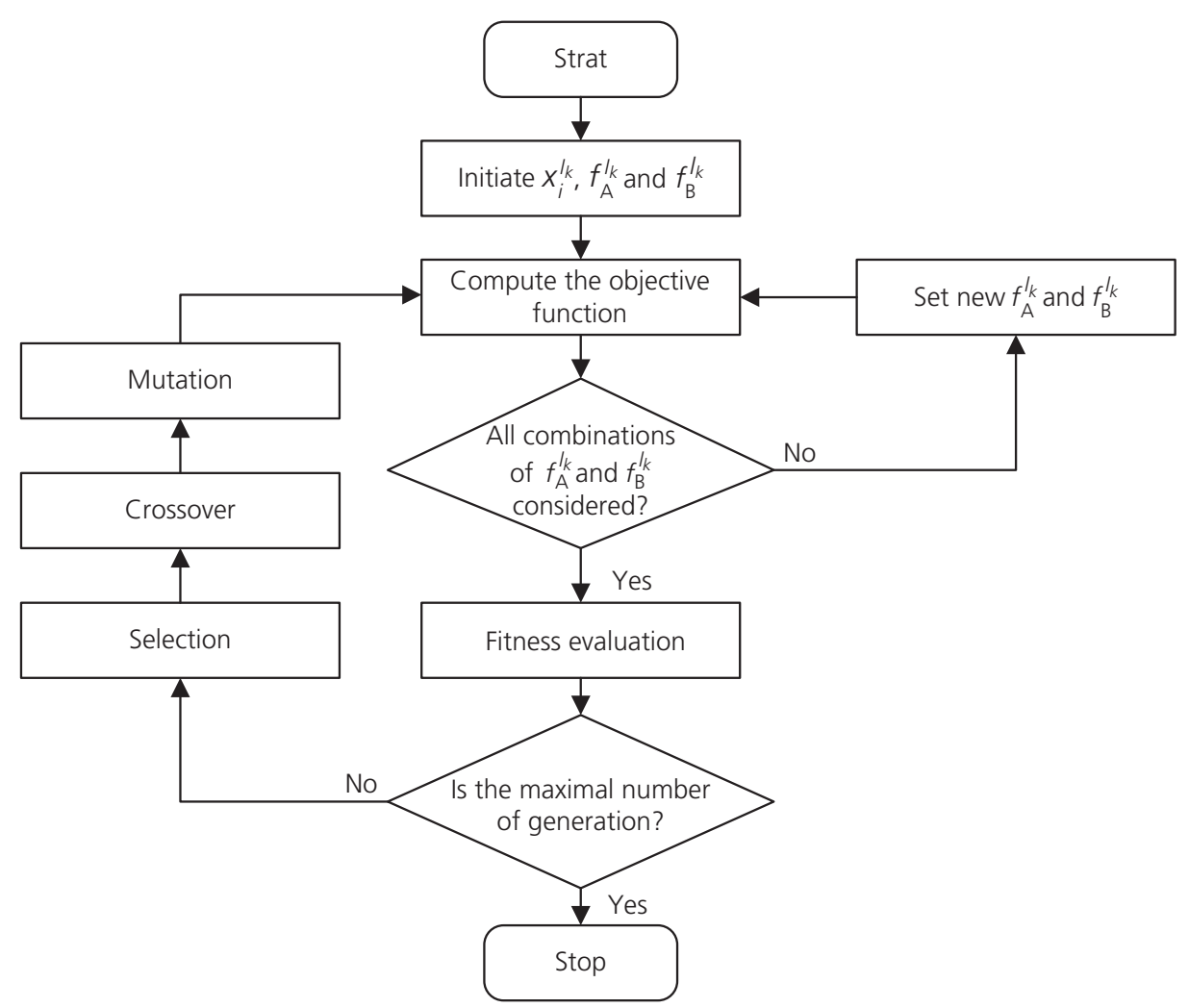

Figure 4. Solution algorithm 
Meanwhile, to yield a minimum cost, an exhaustive search algorithm is used to find the optimal frequencies of different bus routes for each of the chromosomes. The optimal frequencies are subject to Equations 28-30. Figure 4 shows the solution algorithm for the above objective function.

\section{Numerical test}

Now, the methodology proposed in the above section is applied to a branching corridor in Changchun City, China, as shown in Figure 5. The branching corridor is composed of three bus routes: Route 6, running between Dongguang Group and Changchun Railway Station, Route 66 and Route 306. Both Route 66 and Route 306 run between City Hall and Changchun Railway Station. In this branching corridor, there are three feeder lines, and the trunk line is divided into two sections: $L_{1}$ and $L_{2}$. The load profiles of the branching corridor are shown in Figure 6.

According to the study data, when buses serve all stations in a given corridor, the running times of buses operating along routes 66,306 and 6 are 28, 29 and $25 \mathrm{~min}$, respectively, without considering delay stations. The average passenger boarding and alighting times are, respectively, 5.2 and $4.4 \mathrm{~s}$. Time value is assumed to be $\$ 15 /$ pax-h for waiting time, $\$ 10 /$ pax-h for in-vehicle time and $\$ 500 /$ veh-h for running time.
To test the effect of a limited service on the branching corridor, this research calculated the cost of a transit system operating a limited-stop service compared with an all-stop service $\left(x_{i}^{l_{k}}=1\right)$. Figure 7 shows the operational scheme and frequencies of the limited-stop service. In Figure 7, fleet B buses are required to skip the appropriate stations in the trunk-feeder line $\left(l_{1}, l_{2}, l_{3}\right.$, $L_{1}$ and $L_{2}$ ), and the frequencies of both fleet $\mathrm{A}$ and fleet $\mathrm{B}$ are optimised. Table 1 presents the operational results of both the all-stop and limited-stop service. The frequencies and system cost of the all-stop service can be calculated from the above model, if $x_{i}^{l_{k}}=1$. From Table 1, it can be seen that the frequencies of buses are readjusted. This causes the running time to increase, because the total frequencies are changed from 52 to $61 \mathrm{veh} / \mathrm{h}$, while both the passenger waiting time and in-vehicle time drop considerably. Therefore, the service level of the transit system can be improved, and the total cost could be reduced, when compared with the original all-stop service for the branching corridor.

\section{Conclusions}

This paper implements a limited-stop service for a branching corridor to offer optimal bus frequencies. The objective of the paper is to minimise the total cost of a transit system, including the user cost and the operator cost. The objective model can be found by using a trip matrix and the existing data

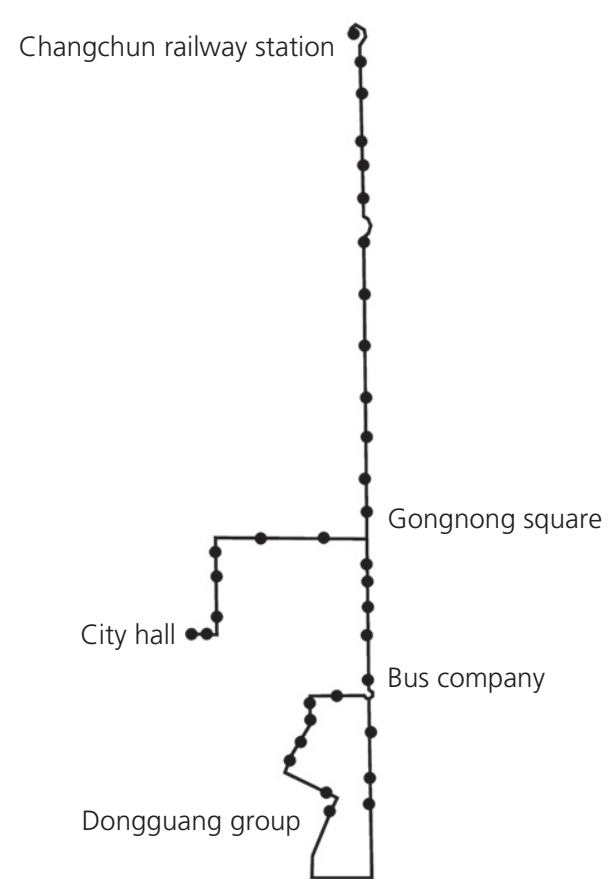

(a)

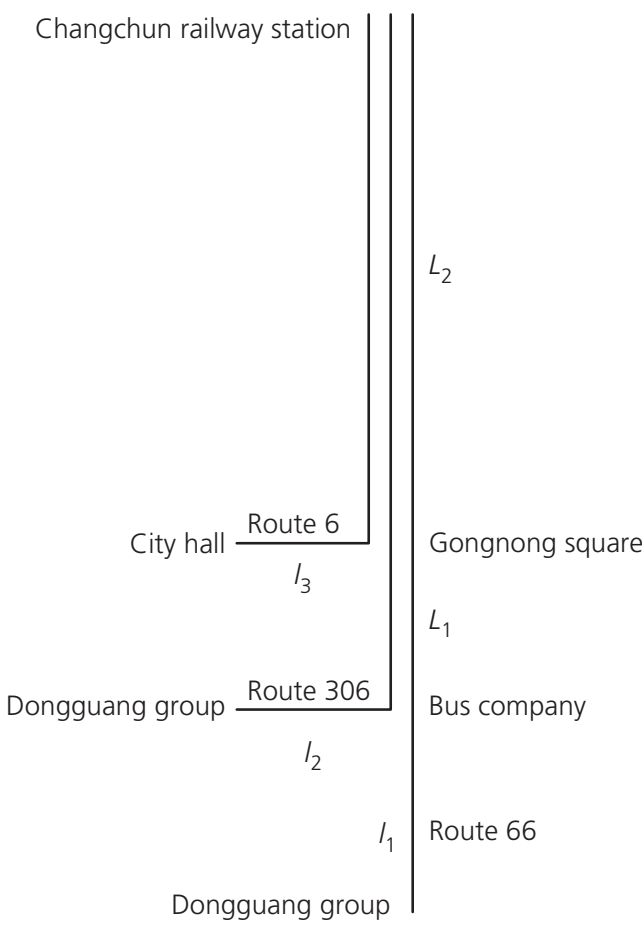

(b)

Figure 5. Branching corridor: (a) street network and (b) existing routes 

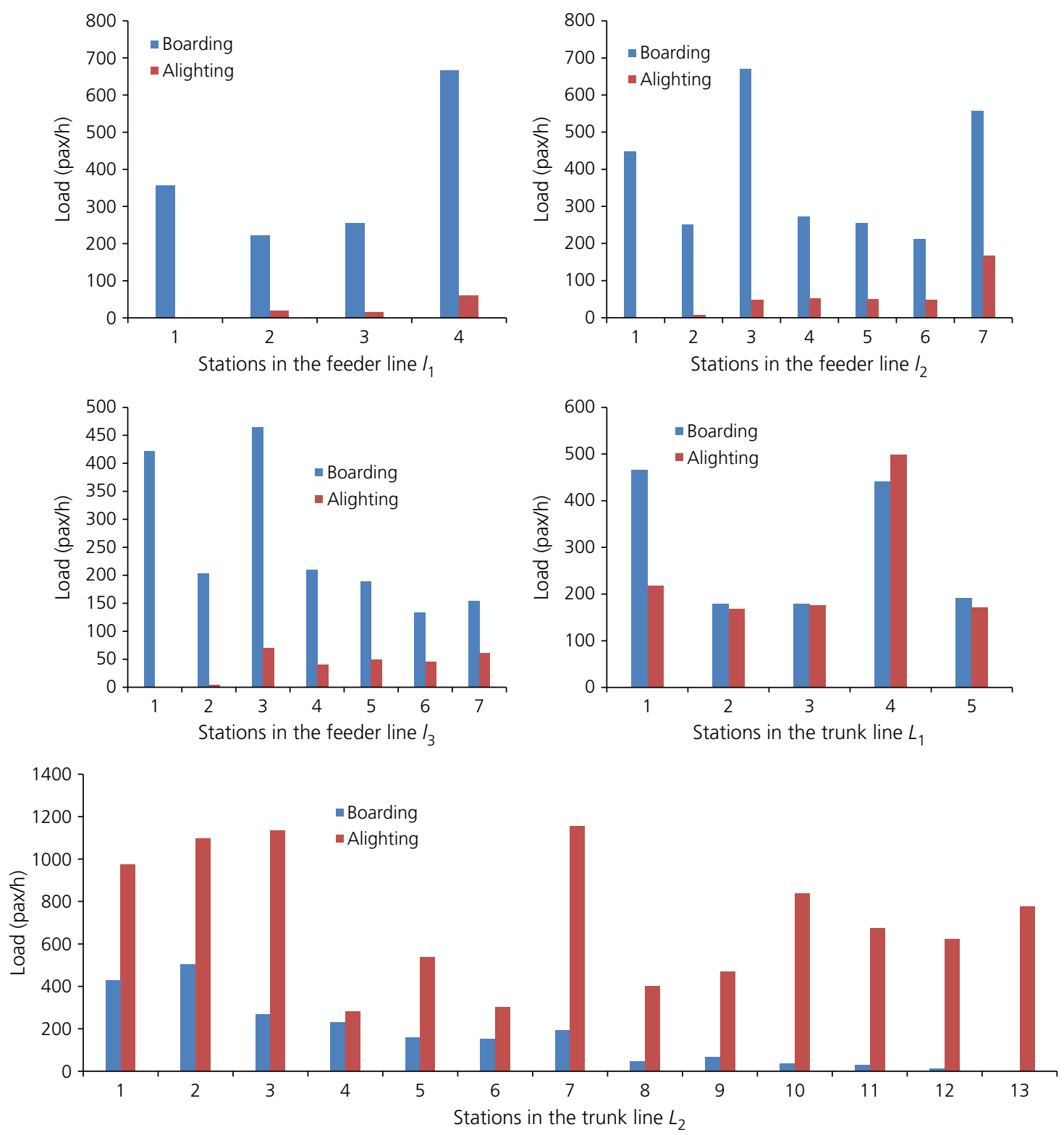

Figure 6. Load profiles of branching corridor

pertaining to bus routes. Since a limited-stop service is different from a traditional all-stop service, certain stations must be designated as limited stops. These are the stations that need to be determined for fleet B. By skipping those specified stations, the limited-stop strategy can offer a high frequency of service at stations which experience high boarding and alighting demands. Meanwhile, the frequency of buses is also optimised.

By comparing the traditional all-stop service and limited-stop service, it can be seen that the passenger waiting time and the in-vehicle time, and the running time of buses can be changed.

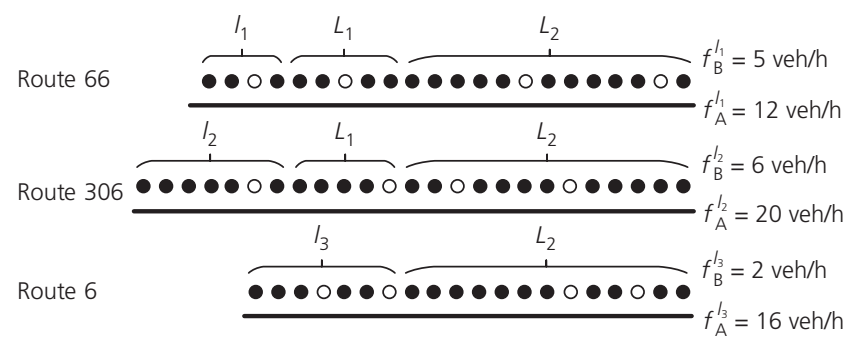

Figure 7. Operational scheme of limited-stop service 
Table 1. Test results

\begin{tabular}{|c|c|c|c|c|c|c|c|c|c|c|}
\hline \multirow[b]{2}{*}{ Types of service } & \multicolumn{3}{|c|}{$f_{A}: v e h / h$} & \multicolumn{3}{|c|}{$f_{\mathrm{B}}: \mathrm{veh} / \mathrm{h}$} & \multirow{2}{*}{$\begin{array}{l}\text { Waiting } \\
\text { time: } \mathrm{h}\end{array}$} & \multirow{2}{*}{$\begin{array}{l}\text { In-vehicle } \\
\text { time: } \mathrm{h}\end{array}$} & \multirow{2}{*}{$\begin{array}{l}\text { Running time } \\
\text { of buses: } h\end{array}$} & \multirow{2}{*}{$\begin{array}{c}\text { Total } \\
\text { cost: } \$ / h\end{array}$} \\
\hline & $I_{1}$ & $I_{2}$ & $I_{3}$ & $I_{1}$ & $I_{2}$ & $I_{3}$ & & & & \\
\hline All-stop service & 13 & 22 & 17 & - & - & - & 423.4559 & 2891 & $43 \cdot 6997$ & 57118 \\
\hline Limited-stop service & 12 & 20 & 16 & 5 & 6 & 2 & $381.0035 \downarrow$ & $2700 \downarrow$ & $47 \cdot 9403 \uparrow$ & $56691 \downarrow$ \\
\hline Change & - & - & - & - & - & - & $-42 \cdot 4524$ & -191 & $4 \cdot 2406$ & -427 \\
\hline
\end{tabular}

$\uparrow$, rise in value; $\downarrow$, decline in value

The waiting time and running time may increase or decrease, based on the optimal frequencies, and the in-vehicle time will be reduced, while the total cost of the transit system will decrease. Therefore, implementing a limited-stop service can reduce the total cost and improve the service level of the transit system. However, because the optimisation method is found using a search algorithm, and because the origin-destination trips of passengers are associated and complicated at different stations, the application ranges of limited-stop services cannot be found. Overcoming this shortcoming would be an interesting project for future research.

\section{Acknowledgement}

The work was supported by the National Natural Science Foundation of China (grant no. 51378237), which collectively funded this project.

\section{REFERENCES}

Afanasiev LL and Liberman SY (1982) Principles for organizing express bus services. Transportation Research Part A: General 17(5): 343-346.

Agrawal J and Mathew TV (2004) Transit route network design using parallel genetic algorithm. Journal of Computing in Civil Engineering 18(3): 248-256.

Baaj MH and Mahmassani HS (1995) Hybrid route generation heuristic algorithm for the design of transit networks. Transportation Research Part C: Emerging Technologies 3(1): 31-50.

Ceder A and Wilson NHM (1986) Bus network design. Transportation Research Part B: Methodological 20(4): 331-344.

Cortés CE, Jara-Díaz S and Tirachini A (2011) Integrating short turning and deadheading in the optimization of transit services. Transportation Research Part A: Policy and Practice 45(5): 419-434, http://dx.doi.org/10.1016/j.tra.2011.02.002.

Dell'Olio L, Ibeas A and Ruisánchez F (2012) Optimizing bus-size and headway in transit networks. Transportation 39(2): 449-464.

Fan W and Machemehl RB (2006) Optimal transit route network design problem with variable transit demand: genetic algorithm approach. Journal of Transportation Engineering 132(1): 40-51.

LeBlanc LJ (1988) Transit system network design. Transportation Research Part B Methodological 22(5): 383-390.

Leiva C, Muñoz JC, Giesen R and Larrain H (2010) Design of limited-stop services for an urban bus corridor with capacity constraints. Transportation Research Part B: Methodological 44(10): 1186-1201.
Mauttone A and Urquhart ME (2009) A route set construction algorithm for the transit network design problem. Computers and Operations Research 36(8): 2440-2449.

Silverman NC (1998) Limited-stop bus service at New York City transit. Journal of Transportation Engineering 124(6): 503-509.

Sivakumaran K, Li Y, Cassidy MJ and Madanat S (2012) Cost-saving properties of schedule coordination in a simple trunk-and-feeder transit system. Transportation Research Part A: Policy and Practice 46(1): 131-139, http://dx.doi.org/10.1016/j.tra.2011.09.013.

Sun A and Mark H (2010) Scheduling considerations for a branching transit route. Journal of Advanced Transportation 38(3): 243-290.

Sun F, Zhu WT, Ye Y and Wang DH (2013) Optimal skip-stop schedule under mixed traffic conditions for minimizing travel time of passengers. Discrete Dynamics in Nature and Society 2013(1857): $1-8$.

Tétreault PR and Ahmed ME (2010) Estimating bus run times for new limited-stop service using archived AVL and APC data. Transportation Research Part A: Policy and Practice 44(6) 390-402, http://dx.doi.org/10.1016/j.tra.2010.03.009.

Yu B, Yang Z and Yao J (2010) Genetic algorithm for bus frequency optimization. Journal of Transportation Engineering 136(6): 576-583

\section{How can you contribute?}

To discuss this paper, please email up to 500 words to the editor at journals@ice.org.uk. Your contribution will be forwarded to the author(s) for a reply and, if considered appropriate by the editorial board, it will be published as discussion in a future issue of the journal.

Proceedings journals rely entirely on contributions from the civil engineering profession (and allied disciplines). Information about how to submit your paper online is available at www.icevirtuallibrary.com/page/authors, where you will also find detailed author guidelines. 